\title{
DYNAMICALLY DEPOSITED THIN-FILM SILICON SOLAR CELLS ON IMPRINTED FOIL USING LINEAR PECVD SOURCES
}

\author{
Jochen Löffler, Maurits C.R. Heijna, Wim J. Soppe and Bas B. Van Aken \\ ECN Solar Energy, P.O. Box 1, 1755 ZG Petten, the Netherlands
}

\begin{abstract}
ECN is developing nip silicon solar cells based on amorphous and microcrystalline thin films on foil. To optimise light trapping we create nanoscale texturisation of the back reflector of the cells by imprinting a UV curable coating layer on the foil. This contribution focuses on i) the suitability of imprinted UV curable coating layers on foil as substrate for thin film Si solar cells; ii) inline PECVD of silicon layers, using linear plasma sources.
\end{abstract}

We show that amorphous silicon solar cells deposited on foil with random texture can achieve the same good light trapping as cells on Asahi U-type glass $\left(\mathrm{J}_{\mathrm{sc}} \sim 15-16\right.$ $\mathrm{mA} / \mathrm{cm}^{2}$ ). Furthermore, we show that a-Si nip cells on foil, processed in dynamic mode in an industrial pilot roll-to-roll system for $30 \mathrm{~cm}$ wide foils, can achieve efficiencies (of over $7 \%$ ) which are only slightly less than for cells made in a UHV lab-scale cluster tool.

Future work will focus on developing and implementing optimised periodic nanotextures for $\mu \mathrm{C}$-Si and micromorph tandem cells and the further development of cells in order to achieve efficiencies of more than $10 \%$ at high deposition rates.

\section{INTRODUCTION}

Roll-to-roll production of thin film Si solar cells has several advantages over batch-type reactor systems, for instance high-throughput fabrication and the application of cheap foil substrates. Flexible, lightweight PV modules gear up to building integrated PV: the most important market for PV in densely populated, developed countries $[1,2]$. Our novel concept for roll-to-roll production of high efficiency nip solar cells is based on amorphous (a-Si:H) and microcrystalline ( $\mu \mathrm{c}-\mathrm{Si}: \mathrm{H})$ silicon thin films on steel foil coated with an insulating barrier layer and sputtered back contact and reflection layer. Furthermore, the barrier layer can be imprinted with any periodic or random texture to increase the light trapping.

Light trapping is most important for microcrystalline Si solar cells and $\mu \mathrm{c}$-Si bottom cells in micromorph tandems, due to the lower absorption of $\mu \mathrm{c}$-Si. To absorb the longer wavelengths (700 nm - $1100 \mathrm{~nm})$, a texture with larger period and height is applied. In the framework of the EU project Silicon-Light [3], we are investigating and will demonstrate the fabrication of ideal periodic structures for light scattering in microcrystalline silicon solar cells and micromorph tandem cells.

\section{SIGNIFICANCE FOR THE FIELD}

The novel concept for roll-to-roll fabrication of thin film silicon solar cells contains a few unique features [4], which offer a great potential for high efficiencies and low cost fabrication. Two of these features are presented and discussed in this paper, i) the suitability of UV curable coating as substrate for thin film Si solar cells and ii) the application of linear plasma sources for the inline deposition of silicon layers.

The standard way to improve the light management of thin film solar cells is to introduce a light scattering structure, either on the front window or at the back reflector. Typically, the growth conditions of the TCO layers are adjusted to get appropriate surface roughness. In contrast, imprinting the UV curable coating layer allows full control of the applied, random or periodic, texture to fully optimise the light trapping.

We have combined in our roll-to-roll PECVD system the previously reported linear symmetric RF sources [5,6], which are excellently suited for deposition of amorphous and microcrystalline doped silicon layers, with a commercially available linear VHF source for the high rate deposition of intrinsic Si absorber layers. The main advantages of linear plasma sources are that only uniformity in a single direction is required and the ease of upscaling the plasma sources to enable deposition on foil substrates of one metre width or more.

\section{EXPERIMENTAL DETAILS}

A UV-curable barrier layer (C-Coatings B.V.) is applied by doctor blading. The texture is imprinted by a PDMS shim in the wet layer and the substrate/layer/shim stack is put through a UV belt oven to harden the lacquer and fixate the texture. Back contacts are sputtered in an AJA lab scale sputter tool, and typically consist of $\sim 250 \mathrm{~nm} \mathrm{Ag}$ and $80 \mathrm{~nm} \mathrm{ZnO}$.

The silicon layer deposition is carried out either in a cluster tool or an inline PECVD system. The cluster tool has three separate UHV chambers for $n$, i and $p$ layer deposition. All chambers have identical flat-plate RF PECVD sources and are closed during deposition to prevent contamination of the transfer chamber and the other chambers. Samples are transported on standard sample holders via a transport arm. 
The inline PECVD system is an industrial pilot roll-to-roll system for foils of width up to $300 \mathrm{~mm}$. The Flexicoat 300 has three inline deposition vacuum chambers. Two chambers are equipped with the previously reported linear symmetric RF $(13.56 \mathrm{MHz})$ sources $[5,6]$, which are excellently suited for deposition of amorphous and microcrystalline doped silicon layers. The intrinsic Si absorber layers are deposited with a linear VHF plasma source $(70 \mathrm{MHz})$. Samples (typically several $10 \times 2.5 \mathrm{~cm}^{2}$ substrates) are fixed to a custom-made sample holder, which is placed in the steel foil that is used as conveyor belt. The vacuum chambers are separated by independently pumped gas sluices to prevent contamination by dopant gasses.

The main advantages of linear plasma sources are that deposition uniformity is only required in one direction, perpendicular to the motion of the substrate(s) and the ease of upscaling the plasma sources to enable deposition on foil substrates of one metre width or more.

The solar cells are defined by the area of the ITO front contact: $4 \times 4 \mathrm{~mm}^{2}$ and $10 \times 10 \mathrm{~mm}^{2}$. For contacting purposes a silver contact pad is e-beamed on the ITO front contact. IV measurements are done on a WACOM sun simulator. The area of the silver contact pad is excluded for the determination of the current density.

\section{RESULTS}

\section{Randomly textured barrier layer}

First, we focus on the texturing of the barrier layer as a means to increase the light trapping. Asahi U-type fluorine-doped tin oxide (FTO) glass is developed for its excellent light trapping properties when used as superstrate for a-Si pin cells, but it is also suitable as substrate for nip solar cells. For this purpose, we made nip cells on Asahi U-type glass, where the FTO, covered with $\mathrm{Ag} / \mathrm{ZnO}$ serves as the back contact. We have used this as reference substrate for comparison with the light scattering of random and periodic textures imprinted in the barrier layer on flexible steel foil.

We have fabricated thin film nip a-Si solar cells on imprinted barrier layers on steel foil. To minimise differences due to back reflection and back contacting properties, both the (textured) barrier layer and the textured FTO of Asahi U-type glass are coated with a reflecting, conducting back contact, in the same $\mathrm{Ag} / \mathrm{ZnO}$ sputtering process. Then, the Si layers on both substrates were processed simultaneously in the lab scale cluster tool. Fig. 1 shows the JV-curve for a imprinted, randomly textured barrier layer, compared with the Asahi reference substrate.

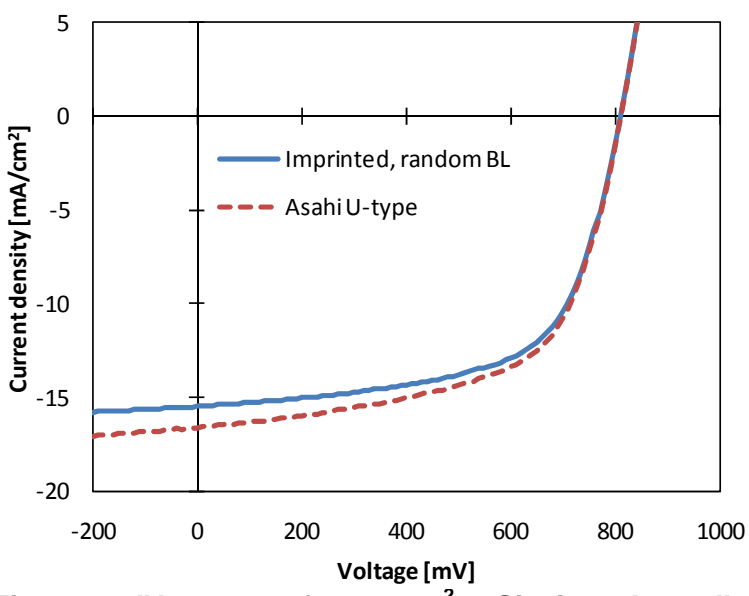

Figure $1 \mathrm{JV}$-curves of $4 \times 4 \mathrm{~mm}^{2}$ a-Si nip solar cells, comparing two rough substrates: Asahi U-type glass (red, broken line) and steel foil with imprinted barrier layer (blue, solid line) and sputtered Ag/ZnO back contact. All layers were processed simultaneously.

As seen in Fig. 1, the a-Si solar cells on imprinted substrates have identical open circuit-voltage $V_{\text {oc }}=810 \mathrm{mV}$ and fill factor $\mathrm{FF}=62 \%$ as nip Si solar cells grown on Asahi U-type glass. The short-circuit current $\mathrm{J}_{\mathrm{sc}}=15 \mathrm{~mA} / \mathrm{cm}^{2}$ is only slightly lower than that of solar cells grown on the Asahi U-type glass $\left(\mathrm{J}_{\mathrm{sc}}=16 \mathrm{~mA} / \mathrm{cm}^{2}\right)$.

The electrical properties of the solar cells are not affected by the barrier layer, as seen in Fig. 1 by the constant open-circuit voltage and fill factor. Therefore, the UVcurable barrier layer is well-suited to be used as imprintable layer to improve the light trapping. However, the short-circuit current, the indicator for the light trapping, is not completely identical to that of the Asahi U-type glass. This is probably due to a combination of not-perfect copying of the texture from master to shim to barrier layer and of using non-ideal masters in the first place.

As next steps, the shape conformity in the imprinting step will could be further improved. To further increase the amount of light trapping towards and beyond that of optimised random textures, like Asahi U-type glass, we will also test periodic textures in the barrier layer that are expected from optical modelling to have an additional increase in light trapping $[7,8]$.

\section{Inline deposition}

The second feature that we present is the use of linear PECVD sources for the inline deposition of Si layers in an industrial pilot roll-to-roll PECVD system. Using the previously described inline PECVD system, we have deposited a-Si nip solar cells on Asahi U-type glass, coated with a sputtered Ag/ZnO back contact. These solar cells are compared with similar cells deposited in a UHV lab scale cluster tool. The resulting JV curves are shown in Fig. 2. 
Comparing the results of the two systems, we observe that the $\mathrm{V}_{\mathrm{oc}}$ of solar cells deposited "dynamically" in the inline PECVD is structurally higher than those of cells fabricated "batch-like" in the cluster tool. In addition, the $\mathrm{J}_{\mathrm{sc}}$ is higher in cluster tool solar cells. This is attributed to variation in the intrinsic absorber layer due to the differences in system and process details. The FF is slightly better in the cluster tool as expected for a UHV system when compared with an industrial pilot system.

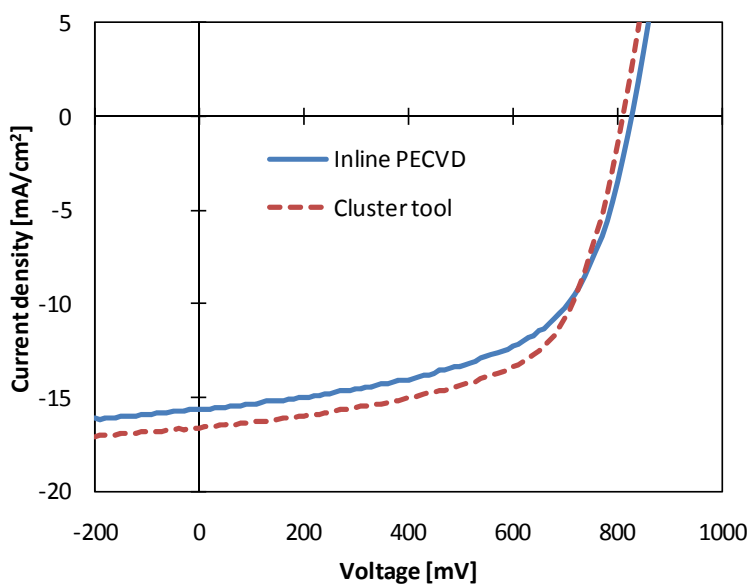

Figure 2. JV-curves of two $4 \times 4 \mathrm{~mm}^{2}$ a-Si nip solar cells, deposited on Asahi U-type glass coated with a sputtered Ag/ZnO back contact, comparing two Si deposition systems: "Cluster tool" is the UHV lab scale system (red, broken line) and "Inline PECVD" the industrial pilot roll-to-roll system (blue, solid line).

The observed combination of higher $\mathrm{V}_{\mathrm{oc}}$ with lower $\mathrm{J}_{\mathrm{sc}}$ in the inline PECVD compared to the values for the cluster tool (see Fig. 2) is related to the differences in the details of the deposition process, such as dynamic vs. static, RF vs. VHF, layout of vacuum chamber, location of gas shower and pumping outlet and different sample holder and substrate heating. observed difference in band gap in the intrinsic absorber layer.

To summarise, the initial efficiency of inline deposited cells is only a few tenths of a percent lower than the cells fabricated in a UHV cluster tool.

\section{Preliminary micromorph solar cells}

We have performed the first preliminary trial depositions of micromorph tandem solar cells. The sub cell thicknesses are 2000 and $240 \mathrm{~nm}$, respectively for the $\mu \mathrm{c}$-Si bottom and the a-Si top cell. In Fig. 3, JV-curves are shown for a set of tandem cells, deposited in the same run on different substrates.

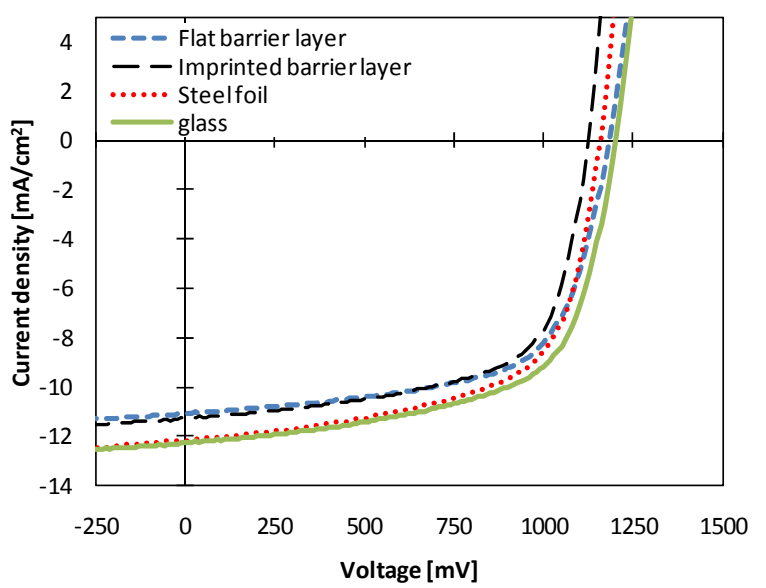

Figure 3. JV-curves of micromorph solar cells on four substrates: steel foil (red, dotted); steel foil with flat, non-textured barrier layer (blue, dashed), idem with random texture (black, long dashed) and standard alkali-free glass (green, solid) and sputtered Ag/ZnO back contact. All layers were processed simultaneously.

Typically, we observe that substrates without barrier layer have a $1 \mathrm{~mA} / \mathrm{cm}^{2}$ higher $\mathrm{J}_{\mathrm{sc}}$ than substrates with barrier layer, but the FF is $2 \%$ higher with barrier layer. The $V_{o c}$ also depends on the substrate with flatter substrates tending to have higher $\mathrm{V}_{\mathrm{oc}}$ than rougher substrates. Note: the applied texture is a random texture, optimised for a-Si solar cells and therefore far from ideal to improve the lighttrapping for $\mu \mathrm{c}$-Si single junction and bottom cells.

The variations in solar cell characteristics for the trial deposition of tandem cells are probably related to the substrate properties. The different substrates (glass / steel foil) and whether or not an (imprinted) barrier layer is applied to the steel foil will have some effect on the details of the layer growth. Small variations in growth rate will lead to variations in bottom cell thickness. Dissimilarities in nucleation and crystal growth will also affect the microcrystalline fraction (distribution) and the $\mu \mathrm{c}-\mathrm{Si} / \mathrm{a}-\mathrm{Si}$ interface. These deviations might again influence the layer growth rate for the a-Si top cell.

Spectral response data for the micromorph tandem cell, a typical a-Si and $\mu \mathrm{c}-\mathrm{Si}$ solar cell are shown in Fig. 4. We observe a higher maximum EQE near $\lambda=500 \mathrm{~nm}$ for the a-Si solar cell, compared to the tandem cell. Also the response between $700-900 \mathrm{~nm}$ for the $\mu \mathrm{c}$-Si solar cell is higher than that of the micromorph solar cell. 




Figure 4. External quantum efficiency for three types of solar cells: micromorph tandem solar cell (green, solid) and single junction a-Si (blue, dashed) and $\mu \mathrm{c}-\mathrm{Si}$ (red, dotted) solar cells.

The lower response at small wavelengths is due to lower thickness of the a-Si absorber layer in the micromorph tandem compared to the single junction a-Si cell. The response at higher wavelengths, $\lambda>900 \mathrm{~nm}$, for the bottom cell is comparable to the response of single junction $\mu \mathrm{c}$-Si solar cells, variations are due to differences in interference fringes from the back reflector.

In summary, the trial tandem fabrication has been rather successful, with efficiencies $>9 \%$ on glass and around $8 \%$ for tandems deposited on steel foil, with or without (imprinted) barrier layer. Further development of micromorph tandem cells will take place, when optimised textures are available.

\section{SUMMARY}

Using linear VHF- and RF-PECVD sources, we have deposited thin film silicon nip solar cells on flexible steel foil substrates. Our UV curable lacquer is well-suited as imprintable barrier layer between the steel foil and the active layers, as the light trapping, measured by the shortcircuit current, is comparable to that of Asahi U-type textured TCO glass.

Furthermore, we show that inline (dynamically) processed a-Si nip cells have efficiencies of over $7 \%$, which is approaching the performance of similar cells made in a UHV lab-scale cluster tool.

Finally, the trial deposition of a micromorph tandem solar cell on steel foil with barrier layer shows a promising initial efficiency of $\sim 8 \%$.

\section{ACKNOWLEDGEMENTS}

This work has been financially supported by the EU (EU FP-7 Energy 2009 - 241477 project "Silicon-Light") and by the Dutch Ministry of Economic Affairs via the ADEM programme.

\section{REFERENCES}

[1] M. Izu and T. Ellison, Sol. En. Mat. Sol. Cells 78 (2003) 613-636.

[2] A. Takano and T. Kamoshita, Jpn. J. Appl. Phys. 43 (2004) 7976.

[3] http://www.silicon-light.eu.

[4] J. Löffler et al., Proc.34th IEEE PVSC (2009).

[5] B.B. Van Aken et al., J. Non-Cryst. Solids 354 (2008) 2392-2396.

[6] B.B Van Aken et al., SPIE Conf. Proc.: Photovoltaic Cell and Module Technologies (2007) pp. 66510C-8.

[7] O. Isabella et al., Proc. $23^{\text {rd }}$ EPVSEC, 2008, pp. 23202323.

[8] B. Lipovsek et al., Proc. $25^{\text {th }}$ EPVSEC, 2010, pp. 31203123. 